\title{
Kinematics and Transmission Performance Analyses of a 2T2R Type 4-DOF Spatial Parallel Manipulator
}

\author{
Haiyan An, ${ }^{1}$ Bin Li $\mathbb{D}^{1,2}$ Shoujun Wang, ${ }^{1,2}$ and Weimin $\mathrm{Ge}^{1,2}$ \\ ${ }^{1}$ Tianjin Key Laboratory for Advanced Mechatronic System Design and Intelligent Control, School of Mechanical Engineering, \\ Tianjin University of Technology, Tianjin 300384, China \\ ${ }^{2}$ National Demonstration Center for Experimental Mechanical and Electrical Engineering Education \\ (Tianjin University of Technology), China
}

Correspondence should be addressed to Bin Li; cnrobot@163.com

Received 11 February 2018; Revised 12 April 2018; Accepted 18 April 2018; Published 24 May 2018

Academic Editor: Shahram Payandeh

Copyright (C) 2018 Haiyan An et al. This is an open access article distributed under the Creative Commons Attribution License, which permits unrestricted use, distribution, and reproduction in any medium, provided the original work is properly cited.

A 2-RPU\&2-SPS spatial parallel mechanism (SPM) is researched. Firstly, the number and property of degrees of freedom (DOF) of the SPM are analyzed by screw theory. There are two rotational and two translational movements (2R2T) of the mechanism that can be achieved. Secondly, the position analyses are researched. For the inverse position analysis, the explicit expression can be obtained from the independent motion parameters of the given mechanism, and the forward position problem is solved by calculating a set of nonlinear equation systems. Then we obtained the workspace of the mechanism based on the analytic formulae of the inverse displacement. Finally, by establishing the Jacobian matrix of the mechanism, the singularity of the mechanism is obtained, and the kinematics transmission performance of the mechanism is studied by using the index of the output efficiency of the limb output of the mechanism. This work will provide the theoretical basis for prototype development and application of the mechanism.

\section{Introduction}

In recent years, with the deepening of the relevant theoretical research of the parallel mechanism and the continuous expansion of the application field, the lower-mobility mechanisms have become the research hotspot in the field of parallel mechanisms. In the comparison between 6-DOF parallel mechanism and lower-mobility mechanism, the latter has the advantages of simpler mechanical design, low manufacturing cost, larger workspace, high accuracy, high velocity, and high stiffness and the accumulated error of positional is little. In the fields of mechanical processing, medical devices, space technology, and sensors and other fields, the lower-mobility mechanism has received increasing attention from industry and academia in various countries.

The 4-DOF parallel mechanism is the most important kind of mechanism in the lower-mobility mechanism, which can be divided into 3T1R, 2T2R, and 3R1T according to the property of DOF. At present, the research depth and breadth of the 4-DOF SPM are far from reaching the research level of 3-DOF SPM. One of the principal reasons is the case that the configuration of the 4-DOF SPM is much smaller than the 3-DOF SPM. In view of this situation, domestic and foreign scholars have carried on the related theoretical research and application to the 4-DOF SPM.

In 1983, Hunt [1] proposed a classical mixed 3-RPS PM, and many literatures could be found about the 3-RPS PM related research work. The kinematic analyses concerning instantaneous motions analysis, dynamic modeling, synthesis, workspace analysis, position analysis, and the like can be found in the literature [2-8]. The 2-DOF PMs of Diamond [9], the 3-DOF PMs of Trivariant [10], Tricept [11], and Delta [12] have been widely applied for industrial treatment. Zeng [13] proposed a series of 2-DOF rotational decoupled PMs based on the mechanism of four-bar linkage. Li [14] researched the type synthesis of the RPR equivalent PM and proposed a new architecture with encouraging potential in practice. Kong [15] firstly used the virtual chain method carried out the PMs type synthesis with a variety of operating modes. The type synthesis of 3-DOF cable driven PMs was also researched by Khakpour [16]. Compared with the 2-DOF or 3-DOF counterparts, the 4-DOF PMs are seldom used in 
practice. Rolland [17] used parallelogram to investigate two 4-DOF parallel mechanisms to eliminate the rotation. Zhao [18] investigated a 4-URU parallel mechanism with the symmetrical topology, which has one rotational DOF and three translational DOFs along the normal of the base. AraujoGómez [19] proposed a two translational and two rotational (2T2R) four-degrees-of-freedom (DOF) parallel kinematic mechanism (PKM) designed as a knee rehabilitation and diagnosis mechatronics system. Q. zhao [20] presents the evolution process of pressure angles from planar parallel mechanisms to spatial parallel mechanisms. L. M. Zhang [21] deals with dynamic dimensional synthesis of the Delta robot using the pressure/transmission angle constraints.

In this paper, a 2T2R 4-DOF 2-RPU \& 2-SPS SPM is investigated and it has a lot of advantages. Firstly, the mechanism moving platform can realize the output of two rotational and two mobile decoupling motions. Secondly, because of the symmetrical arrangement of four moving limbs, the mechanism has a large translational motion space and rotational motion space. Thirdly, the inverse and forward analysis of the mechanism is simple, which simplifies the series of technical problems such as trajectory planning, control, and correction, which are conducive to the application of the mechanism in the field of parallel machine tool equipment. In this paper, firstly, the number and property of degrees of freedom (DOF) of the SPM are analyzed by screw theory. There are two rotational and two translational movements $(2 \mathrm{R} 2 \mathrm{~T})$ of the mechanism that can be achieved. Secondly, the inverse and forward position analyses of the mechanism are researched. Then we obtained the workspace of the mechanism based on the analytic formulae of the inverse displacement. Finally, by establishing the Jacobian matrix of the mechanism, the singularity of the mechanism is obtained, and the kinematics transmission performance of the mechanism is studied by using the index of the output efficiency of the limb output of the mechanism. This work will provide the theoretical basis for prototype development and application of the mechanism.

\section{Structure Characteristics of the 2-RPU\&2-SPS SPM}

As shown in Figure 1, the investigated 4-DOF 2-RㅁU\&2-SPS SPM composed of a moving platform and a base platform and both platforms are attached by two same SPS limbs and two same RPU limbs. The spherical, revolute, prismatic, and universal joints can be abbreviated as $\mathrm{S}, \mathrm{R}, \mathrm{P}$, and $\mathrm{U}$, respectively, and the underlined $\mathrm{p}$ represents actuated joints. Right for the researched SPM, the two same RPU limbs connect the base platform to the moving platform with $\mathrm{R}, \mathrm{P}$, and $U$ joints in sequence. Similarly, two identical SPS limbs connect the base platform to the moving platform by S, P, and $S$ joints in sequence.

In order to establish the position analysis model of the mechanism as shown in Figure 2, place the reference frame $O-x y z$ attached to the base platform with $O$ located in the center of this quadrilateral, and the $x$ axis is collinear with $B_{2} B_{4}$ and points from point $O$ to point $B_{2}$. The $y$ axis is collinear with $B_{1} B_{3}$ and points from point $O$ to point $B_{3}$, and

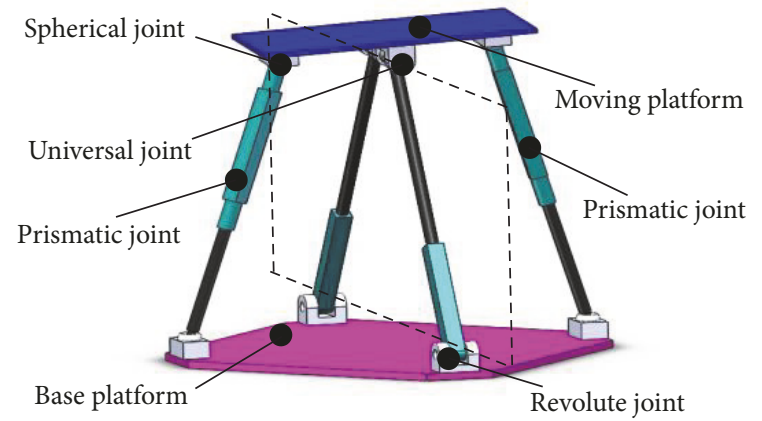

FIGURE 1: CAD model of the proposed 4-DOF SPM 2-RPU\&2-SPS.

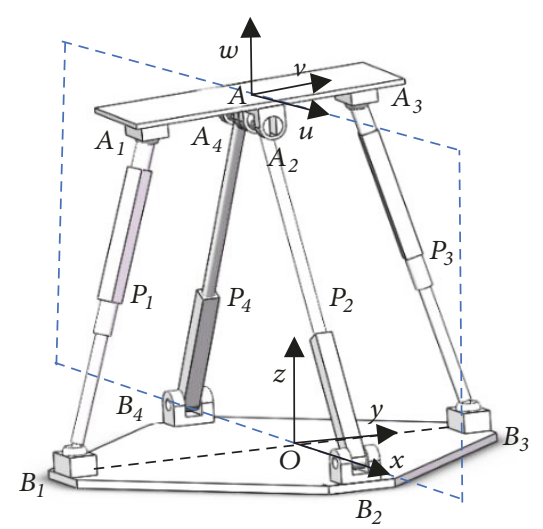

FIGURE 2: Schematic model of the 2-RPU\&2-SPS SPM.

the $z$ axis is perpendicular to the base platform. The moving frame $A-u v w$ is established on the moving platform, where point $A$ is the center of the moving platform, the $u$ axis is collinear with $A_{2} A_{4}$ and points from point A to point $A_{2}$, the $v$ axis is collinear with $A_{1} A_{3}$ and points from point $A$ to point $A_{3}$, and the $w$ axis is perpendicular to the moving platform. Here, $B_{2}$ and $B_{4}$ are the rotational axes of the revolute joints, $A_{1}, A_{3}, B_{1}$, and $B_{3}$ are the centers of the spherical joint, and $A_{2}$ and $A_{4}$, respectively, represent the universal joints center.

Note that, for the RPU limb, the axis of the revolute joint connected to the base platform is parallel to the $y$ axis and the axis of the universal joint connected to the moving platform is also parallel to the $y$ axis. The other axis of the universal joint is parallel to the $u$ axis; the two axes of the universal joints are perpendicular to each other. For the SPS limb, the line connecting the two spherical joints connected to the base platform coincides with the $y$ axis and the connection is symmetrical about the $x$ axis on the base platform. The line connecting the two spherical joints connected to the moving platform coincides with the $v$ axis and the connection is symmetrical about the $u$ axis on the moving platform.

Through the spatial arrangement of the abovementioned kinematic pair, the two RPU limbs can only move in the plane of $x o z$ and the movement of the two SPS limbs can make the moving platform produce a rotation around the $u$ axis from the perspective of geometric constraints. This structure 
determines that the motion trajectory of the moving platform is the motion of a plane and the two rotations of the $v$ axis and the $u$ axis, respectively, thus forming a 2T2R 4-DOF SPM.

In order to research the 2-RPU\&2-SPS SPM conveniently, the moving coordinate system $A-u v w$ is rotated relative to the reference coordinate system $O-x y z$ by $x-z-y$, the corresponding Euler angles of the rotation transformation of each coordinate system are $\psi, \phi$, and $\theta$, and the composition of the rotation matrix is

$$
\begin{aligned}
\mathbf{R} & =\left[\begin{array}{ccc}
c \theta c \phi & s \psi s \theta-c \psi c \theta s \phi & c \psi s \theta+s \psi c \theta s \phi \\
s \phi & c \psi c \phi & -s \psi c \phi \\
-s \theta c \phi & s \psi c \theta+c \psi s \theta s \phi & c \psi c \theta-s \psi s \theta s \phi
\end{array}\right] \\
& =\left[\begin{array}{lll}
u_{x} & v_{x} & w_{x} \\
u_{y} & v_{y} & w_{y} \\
u_{z} & v_{z} & w_{z}
\end{array}\right]=\left[\begin{array}{lll}
\mathbf{u} & \mathbf{v} & \mathbf{w}
\end{array}\right]
\end{aligned}
$$

where sin and cos can be abbreviated to " $s$ " and " $c$ ", respectively, and $\mathbf{u}, \mathbf{v}$, and $\mathbf{w}$ represents the measure of the unit vector of the three coordinate axes of the moving coordinate system in the reference coordinate system.

\section{The DOF Analysis of the 2-RPU\&2-SPS SPM}

The number of DOF of the 4-DOF 2-RPU\&2-SPS SPM can be obtained by the calculation of the criterion Kutzbach Grübler

$$
\begin{aligned}
F & =\lambda(n-g-1)+\sum_{i=1}^{g} f_{i}+v-\zeta \\
& =6 \times(10-12-1)+22+2-2=4
\end{aligned}
$$

where $\lambda$ denotes the task space order, $n$ stands for the links number, $g$ is the kinematic pairs number, $f_{i}$ represents the degrees of freedom of joint $i, v$ denotes the number of redundant constraints, and $\zeta$ represents the local degree of freedom.

We can quickly find the number of DOF of the 2-RPU\&2SPS SPM by using (2). However, it should be pointed out that one shortcoming of the criterion Kutzbach - Grübler is that it can only obtain the number of DOFs of the mechanism rather than indicate the attributes of the DOF, whether they are translational or rotational DOFs. Otherwise, we can analyze the motion of a 2-RPU\&2-SPS SPM via the analysis of the screw theory effectively; then we can easily obtain motions of translation and rotation in three-dimensional space. In Figure 3 through the analysis of the screw theory, the following can be drawn:

$$
\begin{aligned}
& \$_{1}=\left(\begin{array}{llllll}
1 & 0 & 0 & 0 & 0 & 0
\end{array}\right) \\
& \$_{2}=\left(\begin{array}{llllll}
0 & 1 & 0 & 0 & 0 & 0
\end{array}\right) \\
& \$_{3}=\left(\begin{array}{llllll}
0 & 0 & 0 & 0 & -\sin (\alpha) & -\cos (\alpha)
\end{array}\right) \\
& \$_{4}=\left(\begin{array}{llllll}
1 & 0 & 0 & 0 & -q_{2(4)} \cos (\alpha) & q_{2(4)} \sin (\alpha)
\end{array}\right)
\end{aligned}
$$

where $q_{i}=\left\|A_{i} B_{i}\right\|, \quad i=2,4$.

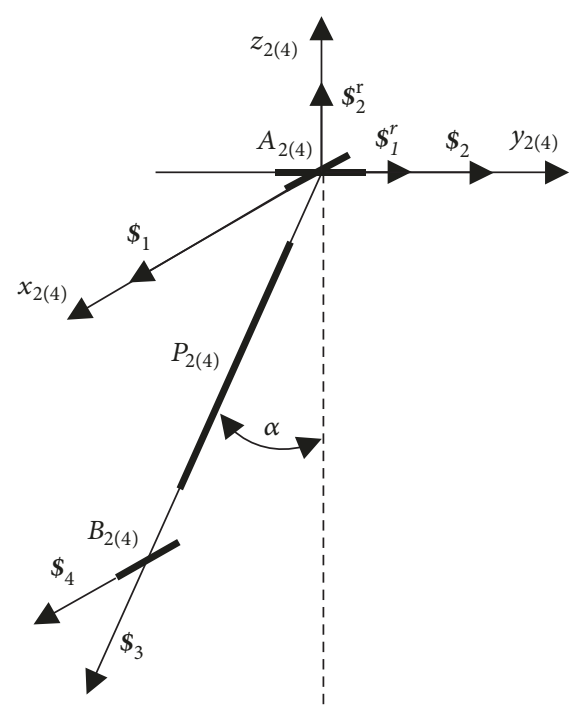

FIgURE 3: Twist system of the RPU limb.

Considering the reciprocity between twist and wrench, the constraint system for the RPU limb can be described as

$$
\begin{aligned}
& \boldsymbol{\$}_{1}^{r}=\left(\begin{array}{llllll}
0 & 1 & 0 & 0 & 0 & 0
\end{array}\right) \\
& \boldsymbol{\$}_{2}^{r}=\left(\begin{array}{llllll}
0 & 0 & 0 & 0 & 0 & 1
\end{array}\right)
\end{aligned}
$$

Similarly, kinematics of screw and reverse screw of the SPS limbs can also be obtained; since the kinematics screw of SPS limb is composed of six linear independence screws without constraint reverse screw, the two SPS limbs do not have constraints on the mechanism.

It can be seen from (4) that a plane along the y axis and a rotation around the $\mathrm{z}$ axis are constrained so that the 2-RPU \& 2-SPS SPM has two translational degrees of freedom (along the $\mathrm{x}$ and $\mathrm{z}$ axes direction) and two rotational degrees of freedom (rotation around the $\mathrm{x}$ and $\mathrm{y}$ axes).

\section{Mechanism Position Analysis}

4.1. Mechanism Analysis of the Inverse Position. As shown in Figure 2, the closed-loop vector equation is constructed, and the position vector $\mathbf{r}=\left(\begin{array}{lll}x & y & z\end{array}\right)^{T}$ of point $\mathrm{A}$ can be represented in the reference coordinate system $O-x y z$ which gives

$$
\mathbf{r}=\mathbf{b}_{i}+q_{i} \mathbf{w}_{\mathrm{i}}-\mathbf{a}_{i} \quad i=1,2,3,4
$$

where $\mathbf{a}_{i}, \mathbf{b}_{i}$ are the position vectors of $A_{i}$ and $B_{i}$, respectively. $\mathbf{w}_{i}$ is the unit vector of $\operatorname{limb} i . \mathbf{a}_{i}=\mathbf{R} \mathbf{a}_{i 0}, \mathbf{R}$ is the rotation matrix of the mechanism, and $\mathbf{a}_{i 0}$ is the measure of $\mathbf{a}_{i}$ in the coordinate system. From the structure of the mechanism, we can see 


$$
\begin{aligned}
& \mathbf{a}_{10}=a_{1}\left(\begin{array}{lll}
0 & -1 & 0
\end{array}\right)^{T}, \\
& \mathbf{a}_{20}=a_{2}\left(\begin{array}{lll}
1 & 0 & 0
\end{array}\right)^{T} \\
& \mathbf{a}_{30}=a_{3}\left(\begin{array}{lll}
0 & 1 & 0
\end{array}\right)^{T}, \\
& \mathbf{a}_{40}=a_{4}\left(\begin{array}{lll}
-1 & 0 & 0
\end{array}\right)^{T} \\
& \mathbf{b}_{10}=b_{1}\left(\begin{array}{lll}
0 & -1 & 0
\end{array}\right)^{T} \\
& \mathbf{b}_{20}=b_{2}\left(\begin{array}{lll}
1 & 0 & 0
\end{array}\right)^{T} \\
& \mathbf{b}_{30}=b_{3}\left(\begin{array}{lll}
0 & 1 & 0
\end{array}\right)^{T}, \\
& \mathbf{b}_{40}=b_{4}\left(\begin{array}{lll}
-1 & 0 & 0
\end{array}\right)^{T}
\end{aligned}
$$

where $a_{1}, a_{2}, a_{3}$, and $a_{4}$, respectively, indicate the distance from point $A$ to points $A_{1}, A_{2}, A_{3}$, and $A_{4}$, and $b_{1}, b_{2}, b_{3}$, and $b_{4}$, respectively, denote the distance from point $B$ to points $B_{1}$, $B_{2}, B_{3}$, and $B_{4}$.

Right for the RPU limb, making the dot product with $\mathbf{c}_{i}$ on both sides of (5) leads to

$$
\left(\mathbf{r}+\mathbf{a}_{i}\right)^{T} \mathbf{c}_{i}=0, \quad i=2,4
$$

where $\mathbf{c}_{2}=\mathbf{c}_{4}=\left(\begin{array}{lll}0 & 1 & 0\end{array}\right)^{T}$.

In the same way, adapting to the SPS limb, making the dot product with $\mathbf{c}_{i}$ on both sides of (5), yields

$$
\left(\mathbf{r}-\mathbf{b}_{i}\right)^{T} \mathbf{c}_{i}=0, \quad i=1,3
$$

where $\mathbf{c}_{1}=\mathbf{R} \mathbf{c}_{10}, \mathbf{c}_{3}=\mathbf{R} \mathbf{c}_{30}$ and $\mathbf{c}_{10}=\mathbf{c}_{30}=\left(\begin{array}{lll}1 & 0 & 0\end{array}\right)$.

As shown in Figure 2, based on the mechanism geometric constraint, the two RPU limbs can just only move in the plane $x o z$, so we can get

$$
u_{y}=0
$$

Through (7), (8), and (9), we can obtain

$$
\begin{aligned}
& \phi=0 \\
& x=z \tan \theta \\
& y=0
\end{aligned}
$$

The position vector $\mathbf{r}=\left(\begin{array}{lll}x & y & z\end{array}\right)^{T}$ for a given reference point $A$ can be determined by (10) for the corresponding attitude angle and the corresponding moving platform relative to the rotation table $R$ of the base platform, and the length of each limb can be obtained by

$$
q_{i}=\left|\mathbf{r}-\mathbf{b}_{i}+\mathbf{a}_{i}\right|, \quad i=1,2,3,4
$$

4.2. Mechanism Analysis of the Forward Position. The relative position of the moving coordinate system in the reference coordinate system is obtained from the given length of the mechanism and a certain mathematical operation. Then according to (11) the forward position analysis of the 2RPUU2-SPSS SPM can be obtained
TABLE 1: Mechanism analysis of the inverse position.

\begin{tabular}{ccccc}
\hline & \multicolumn{5}{c}{ Inputs } \\
Case (a) & $\psi($ deg. $)$ & $\theta($ deg. $)$ & $z(\mathrm{~mm})$ & $x(\mathrm{~mm})$ \\
& 25 & 15 & 650 & 174.1670 \\
& $q_{1}(\mathrm{~mm})$ & $q_{2}(\mathrm{~mm})$ & $q_{3}(\mathrm{~mm})$ & $q_{4}(\mathrm{~mm})$ \\
& 736.8688 & 639.0079 & 844.7550 & 807.5673 \\
\hline & $\psi($ deg. $)$ & $\theta($ deg. $)$ & $z(\mathrm{~mm})$ & $x(\mathrm{~mm})$ \\
Case (b) & 25 & -15 & 650 & -74.1670 \\
& \multicolumn{5}{c}{ Outputs } \\
& $q_{1}(\mathrm{~mm})$ & $q_{2}(\mathrm{~mm})$ & $q_{3}(\mathrm{~mm})$ & $q_{4}(\mathrm{~mm})$ \\
& 736.8688 & 807.5673 & 844.7550 & 639.0079 \\
\hline
\end{tabular}

Then according to (11) the mechanism analysis of the forward position can be obtained as

$$
\begin{aligned}
q_{1}^{2}= & \left(\mathrm{z} * \tan (\theta)-a_{1} * \sin (\psi) * \sin (\theta)\right)^{2} \\
& +\left(b_{1}-a_{1} * \cos (\psi)\right)^{2} \\
& +\left(z-a_{1} * \sin (\psi) * \cos (\theta)\right)^{2} \\
q_{2}^{2}= & \left(\mathrm{z} * \tan (\theta)-b_{2}+a_{2} * \cos (\theta)\right)^{2} \\
& +\left(z-a_{2} * \sin (\theta)\right)^{2} \\
q_{3}^{2}= & \left(\mathrm{z} * \tan (\theta)+a_{3} * \sin (\psi) * \sin (\theta)\right)^{2} \\
& +\left(a_{3} * \cos (\psi)-b_{3}\right)^{2} \\
& +\left(z+a_{3} * \sin (\psi) * \cos (\theta)\right)^{2} \\
q_{4}^{2}= & \left(\mathrm{z} * \tan (\theta)+b_{4}-a_{4} * \cos (\theta)\right)^{2} \\
& +\left(z+a_{4} * \sin (\theta)\right)^{2}
\end{aligned}
$$

Since the positive solution constrained equations of the 4-DOF SPM are complex, this paper will directly call the mathematical calculation software to solve the numerical solution of the nonlinear Equations (12)-(15).

4.3. Numerical Examples. According to the forward and inverse position analysis of Sections 4.1 and 4.2 , the selections of the mechanism parameters are $a_{1}=a_{3}=150 \mathrm{~mm}, a_{2}=a_{4}$ $=75 \mathrm{~mm}, b_{1}=b_{3}=550 \mathrm{~mm}, b_{2}=b_{4}=350 \mathrm{~mm}$. Right for the inverse position analysis, the calculated output results are shown in Table 1 and Figure 4 by calculating two given different sets of inputs ( $\left.\begin{array}{lll}\psi & \theta & z\end{array}\right)$. Adapting to the mechanism analysis of the forward position, for the purpose of testifying the correlation of the analyses of the inverse and forward position, the outputs value of the inverse position analysis are served as the inputs $\left(\begin{array}{llll}q_{1} & q_{2} & q_{3} & q_{4}\end{array}\right)$ of the forward position analysis, and the output results can be obtained by solving the nonlinear equation system as shown in Table 2 and Figure 5. 

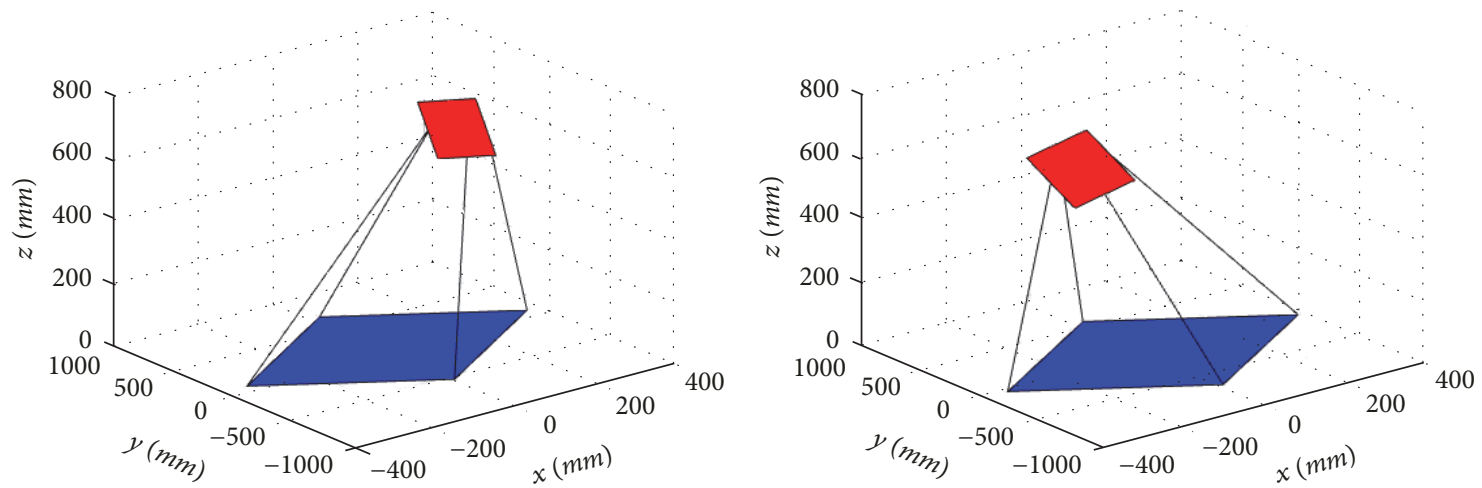

FIgURE 4: Different configurations for inverse position.
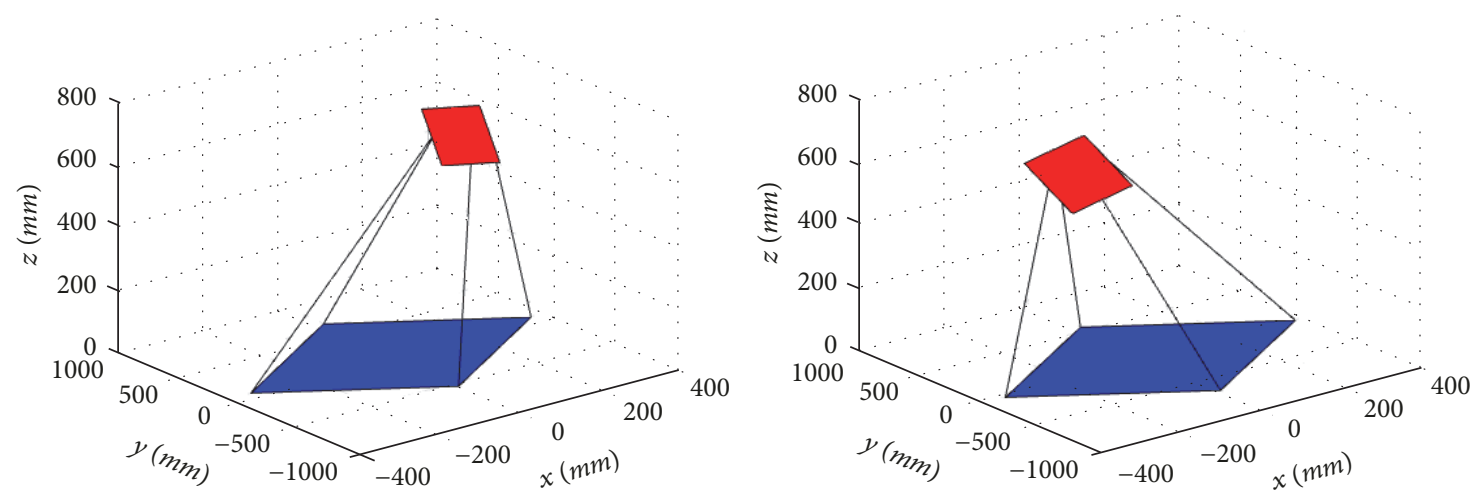

FiguRE 5: Different configurations for forward position.

TABLE 2: Mechanism analysis of the forward position.

\begin{tabular}{ccccc}
\hline & \multicolumn{4}{c}{ Inputs } \\
Case (a) & $q_{1}(\mathrm{~mm})$ & $q_{2}(\mathrm{~mm})$ & $q_{3}(\mathrm{~mm})$ & $q_{4}(\mathrm{~mm})$ \\
& 736.8688 & 639.0079 & 844.7550 & 807.5673 \\
& $\psi($ deg. $)$ & $\theta$ (deg.) & $z(\mathrm{~mm})$ & $x(\mathrm{~mm})$ \\
& 25 & 15 & 650 & 174.1670 \\
\hline \multirow{5}{c}{ Outputs } \\
Case (b) & $q_{2}(\mathrm{~mm})$ & $q_{2}(\mathrm{~mm})$ & $q_{3}(\mathrm{~mm})$ & $q_{4}(\mathrm{~mm})$ \\
& 736.8688 & 807.5673 & 844.7550 & 639.0079 \\
& $\psi($ deg.) & \multicolumn{4}{c}{ Outputs } \\
& 25 & \multicolumn{4}{c}{-15} & $z(\mathrm{~mm})$ & $x(\mathrm{~mm})$ \\
& \multicolumn{5}{c}{650} \\
\hline
\end{tabular}

As shown in Figures 4 and 5, we can see that the output of the positive and inverse solution of the mechanism is consistently, which proves the accuracy of the calculation.

\section{The Overall Jacobian Matrix}

5.1. The Overall Jacobian Matrix of the Mechanism. Equation (5) at time differentiating can be written as

$$
\dot{\mathbf{r}}=\dot{q}_{i} \mathbf{w}_{i}+\boldsymbol{\omega}_{i} \times q_{i} \mathbf{w}_{i}-\boldsymbol{\omega} \times \mathbf{a}_{i} \quad i=1,2,3,4
$$

where " $x$ " represents the cross product of the vectors, $\dot{q}_{i}$ denotes the velocity of the linear actuator $i$, and $\mathbf{w}_{i}$ stands for the angular velocity of link, and $A_{i} B_{i}, \dot{\mathbf{r}}=\left(\begin{array}{lll}\dot{x} & \dot{y} & \dot{z}\end{array}\right)^{T}$, and $\boldsymbol{\omega}=$ $\left(\begin{array}{lll}\omega_{x} & \omega_{y} & \omega_{z}\end{array}\right)$, respectively, stand for the spatial linear velocity and the spatial angular velocity of the moving platform.

Through dot multiplying on both sides of (16) with $\mathbf{w}_{i}$, the passive variables $\mathbf{w}_{i}$ are eliminated; this leads to

$$
\dot{q}_{i}=\dot{\mathbf{w}}_{i} \dot{\mathbf{r}}+\boldsymbol{\omega}\left(\mathbf{a}_{i} \times \mathbf{w}_{i}\right) \quad i=1,2,3,4
$$

When the SPM is not in a singular configuration, (17) can be expressed as

$$
\dot{\mathbf{q}}_{a}=\mathbf{J}_{a} \dot{\mathbf{X}}
$$


where

$$
\begin{aligned}
\dot{\mathbf{q}}_{a} & =\left[\begin{array}{l}
\dot{q}_{1} \\
\dot{q}_{2} \\
\dot{q}_{3} \\
\dot{q}_{4}
\end{array}\right], \\
\dot{\mathbf{X}} & =\left[\begin{array}{c}
\dot{\mathbf{r}} \\
\mathbf{\omega}
\end{array}\right], \\
\mathbf{J}_{a} & =\left[\begin{array}{cc}
\mathbf{w}_{1}^{T} & \left(\mathbf{a}_{1} \times \mathbf{w}_{1}\right)^{T} \\
\mathbf{w}_{2}^{T} & \left(\mathbf{a}_{2} \times \mathbf{w}_{2}\right)^{T} \\
\mathbf{w}_{3}^{T} & \left(\mathbf{a}_{3} \times \mathbf{w}_{3}\right)^{T} \\
\mathbf{w}_{4}^{T} & \left(\mathbf{a}_{4} \times w_{4}\right)^{T}
\end{array}\right]
\end{aligned}
$$

Equation (18) denotes the solution of the inverse velocity for the 2-RPU\&2-SPS SPM.

Equations (7) and (8) at time differentiating, respectively, lead to

$$
\begin{aligned}
& \mathbf{c}_{i} \cdot \dot{\mathbf{r}}+\left(\mathbf{c}_{i} \times \mathbf{a}_{i}\right) \cdot \boldsymbol{\omega}=0 \quad i=2,4 \\
& \mathbf{c}_{i} \cdot \dot{\mathbf{r}}+\left[\mathbf{c}_{i} \times\left(\mathbf{r}-\mathbf{b}_{i}\right)\right] \cdot \boldsymbol{\omega}=0 \quad i=1,3
\end{aligned}
$$

When the mechanism is no singularity, (20) and (21) can be expressed as

$$
\mathbf{0}=\mathbf{J}_{c} \dot{\mathbf{X}}
$$

where $\mathbf{J}_{c}=\left[\begin{array}{cc}\mathbf{c}_{1}^{T} & {\left[\mathbf{c}_{1} \times\left(\mathbf{r}-\mathbf{b}_{1}\right)\right]^{T}} \\ \boldsymbol{c}_{2}^{T} & \left(\mathbf{c}_{2} \times \mathbf{a}_{2}\right)^{T} \\ \boldsymbol{c}_{3}^{T} & {\left[\mathbf{c}_{3} \times\left(\mathbf{r}-\mathbf{b}_{3}\right)\right]^{T}} \\ \boldsymbol{c}_{4}^{T} & \left(\mathbf{c}_{4} \times \mathbf{a}_{4}\right)^{T}\end{array}\right]$ and 0 represents a zero matrix of $4 \times 1$.

Simultaneously to (18) and (22) we can get

$$
\dot{\mathbf{q}}=\mathrm{J}_{0} \dot{\mathbf{X}}
$$

where $\dot{\mathbf{q}}=\left[\begin{array}{c}\dot{\mathbf{q}}_{a} \\ 0\end{array}\right], \mathbf{J}_{0}=\left[\begin{array}{l}\mathbf{J}_{a} \\ \mathbf{J}_{c}\end{array}\right]$ is the overall Jacobian matrix of the 2-RPU\&2-SPS SPM.

Equation (10) at time differentiating can be written as

$$
\begin{aligned}
& \dot{\phi}=0 \\
& \dot{x}=\dot{z} \tan (\theta)+z \sec ^{2}(\theta) \dot{\theta} \\
& \dot{y}=0
\end{aligned}
$$

The constrained Jacobian matrix of the2-Rㅁ&2-SP्PS SPM is

$$
\mathbf{J}_{0}=\left[\begin{array}{llll}
J_{11} & J_{12} & J_{13} & J_{14} \\
J_{21} & J_{22} & J_{23} & J_{24} \\
J_{31} & J_{32} & J_{33} & J_{34} \\
J_{41} & J_{42} & J_{43} & J_{44}
\end{array}\right]
$$

where

$$
\begin{aligned}
& J_{11}=\frac{x-a_{1} \sin (\psi) \sin (\theta)}{L_{1}} \\
& J_{12}=\frac{z-a_{1} \sin (\psi) \cos (\theta)}{L_{1}} \\
& J_{13}=\frac{z a_{1} \sin (\psi) \sin (\theta)-a_{1} x \sin (\psi) \cos (\theta)}{L_{1}} \\
& J_{14} \\
& =\frac{-a_{1} x \cos (\psi) \sin (\theta)+a_{1} b_{1} \sin (\psi)-a_{1} z \cos (\psi) \cos (\theta)}{L_{1}} \\
& J_{21}=\frac{x-b_{2}+a_{2} \cos (\theta)}{L_{2}}, \\
& J_{22}=\frac{z-a_{2} \sin (\theta)}{L_{2}} \\
& J_{23}=\frac{-a_{2} x \sin (\theta)+a_{2} b_{2} \sin (\theta)-a_{2} z \cos (\theta)}{L_{2}} \\
& J_{24}=0 \\
& J_{31}=\frac{x+a_{3} \sin (\psi) \sin (\theta)}{L_{3}} \\
& J_{32}=\frac{z+a_{3} \sin (\psi) \cos (\theta)}{L_{3}} \\
& J_{33}=\frac{-z a_{3} \sin (\psi) \sin (\theta)+a_{3} x \sin (\psi) \cos (\theta)}{L_{3}} \\
& J_{34}=\frac{a_{3} x \cos (\psi) \sin (\theta)+a_{3} b_{3} \sin (\psi)+a_{3} z \cos (\psi) \cos (\theta)}{L_{3}} \\
& J_{41}=\frac{x+b_{4}-a_{4} \cos (\theta)}{L_{4}}, \\
& J_{42}=\frac{z+a_{4} \sin (\theta)}{L_{4}} \\
& J_{43}=\frac{a_{4} x \sin (\theta)+a_{4} b_{4} \sin (\theta)+a_{4} z \cos (\theta)}{L_{4}} \\
& J_{44}=0 \\
& L_{1}=\left(z^{2} \sec ^{2}(\theta)+a_{1}^{2}+b_{1}^{2}-2 a_{1} z \sin (\psi) \sec (\theta)\right. \\
& \left.-2 a_{1} b_{1} \cos (\psi)\right)^{1 / 2} \\
& L_{2}=\left(z^{2} \sec ^{2}(\theta)+a_{2}^{2}+b_{2}^{2}-2 b_{2} z \tan (\theta)-2 a_{2} b_{2} \cos (\theta)\right)^{1 / 2} \\
& L_{3}=\left(z^{2} \sec ^{2}(\theta)+a_{3}^{2}+b_{3}^{2}+2 a_{3} z \sin (\psi) \sec (\theta)\right. \\
& \left.-2 a_{3} b_{3} \cos (\psi)\right)^{1 / 2} \\
& L_{4}=\left(z^{2} \sec ^{2}(\theta)+a_{4}^{2}+b_{4}^{2}+2 b_{4} z \tan (\theta)-2 a_{4} b_{4} \cos (\theta)\right)^{1 / 2}
\end{aligned}
$$

In combination with the geometric constraints of the 2RPU\&2-SPS SPM, the input parameters of the mechanism are set as $-40^{\circ} \leqslant \psi \leqslant 40^{\circ},-40 \leqslant \theta \leqslant 40^{\circ}$ and the overall Jacobian of the mechanism is shown in Figure 6. 


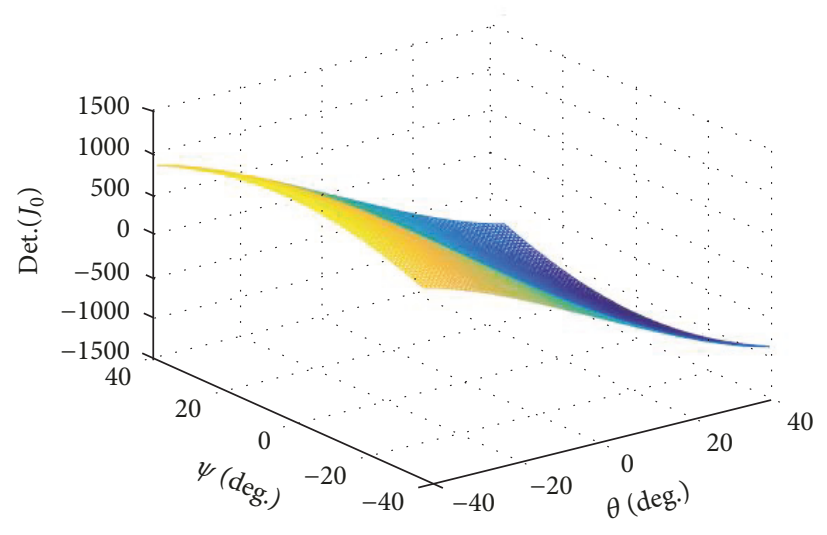

Figure 6: The overall Jacobian diagram of the mechanism.

5.2. Singularity Analysis. Singular configuration is in the process of the parallel mechanism operation to achieve a state of special configuration; under this kind of posture or nearby, mechanism can get out of control, mainly for mechanism freedom change; stiffness and transmission performance are reduced, which more serious when it causes the damage of organization structure, so the singularity is necessary.

(1) When $|A| \neq 0$ and $|D|=0$, which means that the determinant value of the input coefficient matrix is 0 , then, the mechanism kinematic limb is in a boundary singular positon. That is, the output vector corresponding to a nonzero input vector is 0 , so no velocity vector is generated at the output. In this configuration, the output element will lose one or more degrees of freedom while resisting one or more forces or moments when no torque is applied to the input.

(2) When $|A|=0$ and $|D| \neq 0$, that is, when the determinant value of the output coefficient matrix is 0 , the corresponding mechanism reaches a singular position in the workspace, which is called actuated singularity of the mechanism. In other words, when all the actuated elements are locked, the output elements of the mechanism can still be partially moved. In this configuration, the output element will gain one or more degrees of freedom while being unable to resist one or more forces or moments when the input is locked.

(3) When $|A|=0$ and $|D|=0$, the input-output coefficient matrix determinant value is also 0 . If the mechanism result parameter satisfies certain specific conditions and the positional relationship reached by the limb satisfies the kinematics equation configuration, the configuration of the mechanism is called hybrid singularity. This singularity is characterized by the ability of the limb to withstand limited movement when the actuated element is locked or the limited input does not produce any output.
Equation (23) shows the relation of the singularity configuration of the mechanism:

$$
A \dot{\mathbf{X}}+D \dot{\mathbf{q}}_{i}=0
$$

where

$$
\begin{aligned}
& A=\left[\begin{array}{ccccc}
J_{11}\left|L_{1}\right| & J_{12}\left|L_{1}\right| & J_{13}\left|L_{1}\right| & J_{14}\left|L_{1}\right| \\
J_{21}\left|L_{2}\right| & J_{22}\left|L_{2}\right| & J_{23}\left|L_{2}\right| & J_{24}\left|L_{2}\right| \\
J_{31}\left|L_{3}\right| & J_{32}\left|L_{3}\right| & J_{33}\left|L_{3}\right| & J_{34}\left|L_{3}\right| \\
J_{41}\left|L_{4}\right| & J_{42}\left|L_{4}\right| & J_{43}\left|L_{4}\right| & J_{44}\left|L_{4}\right|
\end{array}\right] \\
& D=-\left[\begin{array}{cccc}
\left|L_{1}\right| & 0 & 0 & 0 \\
0 & \left|L_{2}\right| & 0 & 0 \\
0 & 0 & \left|L_{3}\right| & 0 \\
0 & 0 & 0 & \left|L_{4}\right|
\end{array}\right]
\end{aligned}
$$

A is the output coefficient matrix. D is the input coefficient matrix.

According to (27), the singularity configuration of the mechanism may be obtained:

(1) Because the length of the limb is not zero, there is no configuration singularity and hybrid singularity.

(2) When $|A|=0,|D| \neq 0$, there is an actuated singularity.

When

$$
|A|=\left|\begin{array}{lllll}
J_{11}\left|L_{1}\right| & J_{12}\left|L_{1}\right| & J_{13}\left|L_{1}\right| & J_{14}\left|L_{1}\right| \\
J_{21}\left|L_{2}\right| & J_{22}\left|L_{2}\right| & J_{23}\left|L_{2}\right| & J_{24}\left|L_{2}\right| \\
J_{31}\left|L_{3}\right| & J_{32}\left|L_{3}\right| & J_{33}\left|L_{3}\right| & J_{34}\left|L_{3}\right| \\
J_{41}\left|L_{4}\right| & J_{42}\left|L_{4}\right| & J_{43}\left|L_{4}\right| & J_{44}\left|L_{4}\right|
\end{array}\right|=0
$$

there is an actuated singularity, then we obtain $\theta=77.63^{\circ}$ or $0^{\circ}$.

5.3. Velocity and Acceleration. Simultaneous (23) and (24) can obtain the spatial linear velocity and the spatial angular velocity of the moving platform.

$$
\begin{aligned}
\boldsymbol{\omega} & =\left[\begin{array}{lll}
\omega_{x} & \omega_{y} & \omega_{z}
\end{array}\right]^{T}=\left[\begin{array}{llll}
\dot{\psi} c \theta & \dot{\theta} & -\dot{\psi} s & s
\end{array}\right]^{T} \\
\dot{\mathbf{r}} & =\left[\begin{array}{lll}
\dot{x} & \dot{y} & \dot{z}
\end{array}\right]^{T} \\
& =\left[\begin{array}{llll}
\dot{z} * \tan (\theta)+z *\left(\sec ^{2}(\theta)\right) * \dot{\theta} & 0 & 1
\end{array}\right]^{T}
\end{aligned}
$$

Differentiating (30) and (31) with respect to time, respectively, can obtain the spatial linear acceleration and the spatial angular acceleration of the moving platform.

$$
\begin{aligned}
& \ddot{\mathbf{r}}=\left[\ddot{z} * \tan (\theta)+2 * \dot{z} * \sec ^{2}(\theta) * \dot{\theta}+z * \sec ^{2}(\theta) * \ddot{\theta}+2 * z *(\dot{\theta})^{2} * \sec ^{2}(\theta) * \tan (\theta) \quad 0 \quad 0\right]^{T} \\
& \dot{\boldsymbol{\omega}}=[\ddot{\psi} * \cos (\theta)-\dot{\psi} * \sin (\theta) * \dot{\theta} \ddot{\theta}-\ddot{\psi} * \sin (\theta)-\dot{\psi} * \cos (\theta) * \dot{\theta}]^{T}
\end{aligned}
$$




\section{Workspace Analysis}

The working space of the parallel mechanism is one of the indexes of the comprehensive performance of the mechanism. It directly reflects the working ability of the mechanism, and its analysis results provide a theoretical basis for the design and application of the 2-RPU\&2-SPS SPM. In Section 3, based on the position analysis, the working space of the SPM is obtained by utilizing the search algorithm combined with the structural characteristics of 2-RPU\&2-SPS SPM, the inverse kinematic solution, and the limitation of the limb length.

6.1. Constraints on Workspace. The parallel mechanism moving platform and base platform are relate to the limb, which needs to use the spherical joint and revolute joint; then the conditions of the spherical joint and revolute joint angle range need to be set.

(1) Limit of Limb

$$
q_{i \min } \leq q_{i} \leq q_{i \max } \quad i=1,2,3,4
$$

where $q_{i}$ represents the length of $\operatorname{limb} i, q_{i \min }$ denotes the minimum length of limb $i$, and $q_{i \max }$ stands for the maximum length of limb $i$.

\section{(2) Limit of Rotation Angle}

$$
\theta_{i \min } \leq \theta_{i} \leq \theta_{i \max }
$$

where $\theta_{i}$ represents the angle of revolute joint $i, \theta_{i \text { min }}$ denotes the minimum angle of revolute joint $i$, and $\theta_{i \max }$ stands for the maximum angle of revolute joint $i$.

$$
\psi_{i \min } \leq \psi_{i} \leq \psi_{i \max }
$$

where $\psi_{i}$ represents the angle of revolute joint $i, \psi_{i \text { min }}$ denotes the minimum angle of revolute joint $i$, and $\psi_{i \max }$ stands for the maximum angle of revolute joint $i$.

6.2. Workspace Analysis. According to the structural characteristics of the 2-RPU \& 2-SPS SPM, the scale parameters of the mechanism are selected as $a_{1}=a_{3}=150 \mathrm{~mm}, a_{2}=a_{4}$ $=75 \mathrm{~mm}, b_{1}=b_{3}=550 \mathrm{~mm}$, and $b_{2}=b_{4}=350 \mathrm{~mm}$, and the constraints of the mechanism are $-30^{\circ} \leq \psi_{i} \leq 30^{\circ}$, $-45^{\circ} \leq \theta_{i} \leq 45^{\circ}$, and $700 \leq q_{i} \leq 1000$, and the working space is shown in Figure 7.

Because the 2-RPU\&2-SPS SPM can only move along the $x$ and $z$ axes in the $x o z$ plane and the rotation of the $x$ and $y$ axes, the working space of the mechanism in the $x o z$ plane is about the symmetric distribution of the $z$ axis in Figure 8, and the working space is roughly in the range of $-410 \leq x \leq 410(\mathrm{~mm})$ and $655 \leq z \leq 860(\mathrm{~mm})$; compared to the scale parameters of the mechanism, the work space of the mechanism is larger. The workspaces of the mechanism in xoy plane and yoz plane are shown in Figure 9 and Figure 10.

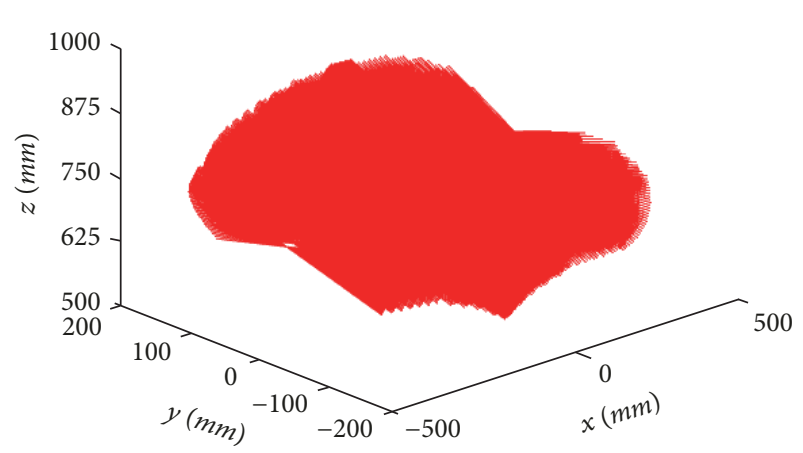

FIGURE 7: The entire workspace of the mechanism.

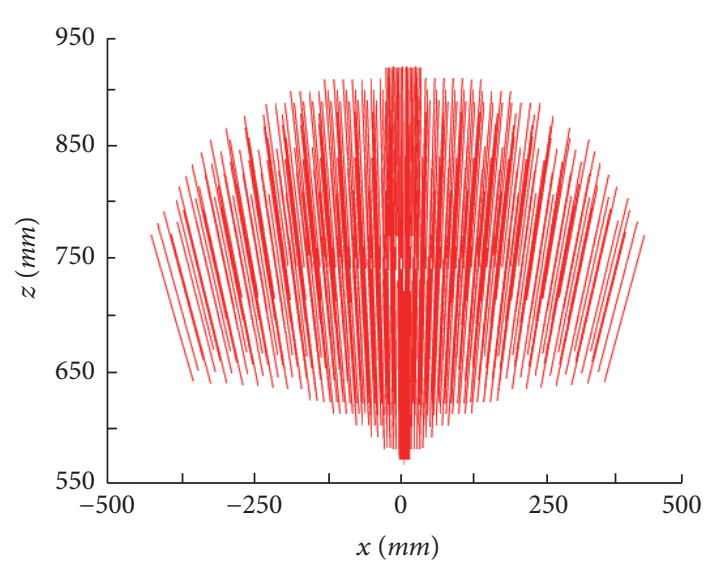

FIGURE 8: The workspace of the mechanism in $x-z$.

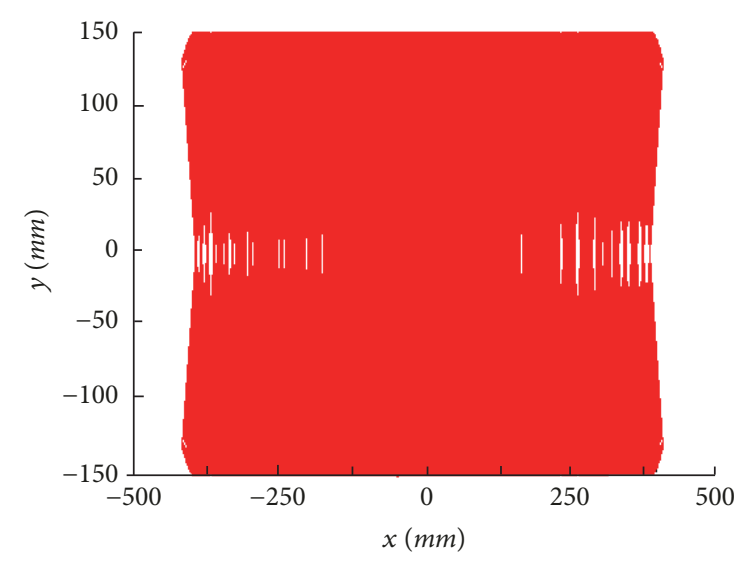

FIGURE 9: The workspace of the mechanism in $x-y$.

\section{The Analysis of the Transmission Performance}

One of the most important factors in designing and analyzing of the 2-RPU\&2-SPS SPM is the evaluation of the performance, and there are many indicators of performance evaluation, such as dexterity, transmission angle, and torque/force transmission performance. Traditional transmission pressure angle is usually used to assess the limb of a single loop and 


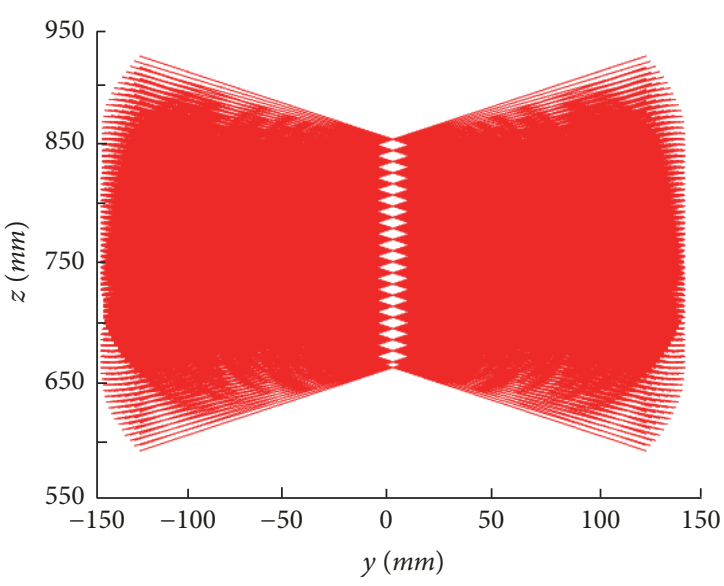

FIGURE 10: The workspace of the mechanism in $\mathrm{y}-\mathrm{z}$.

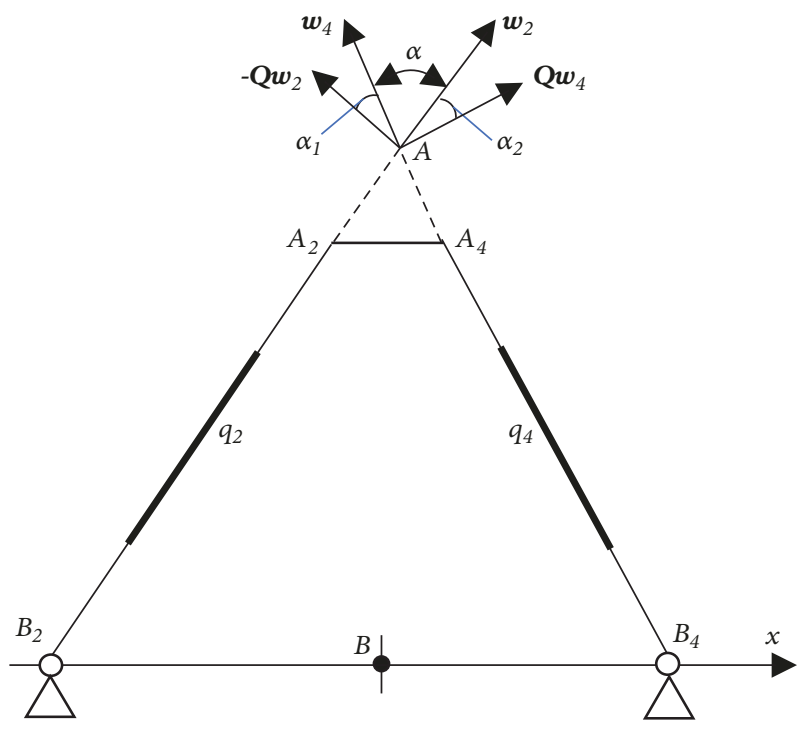

FIGURE 11: Pressure angles between RPU limbs.

should not be utilized for the evaluation of spatial parallel mechanism. However, in this paper, the 2-RPU\&2-SPS SPM with two identical RPU limbs and two identical SPS libms and the two RPU limbs move in the same plane, due to the these two rotating joint axes being parallel to each other. Based on the special structure of the research mechanism, the pressure angle can be served to evaluate the transmission performance of the SPM torque/force.

The analysis of the 2-RPU\&2-SPS SPM transmission performance in this paper is based on the pressure angle of the limbs, and the definition of the pressure angle is shown in Figures 11 and 12.

Right for the RPU limb, the acute angle $\alpha_{i}$ shown in Figure 11 is angle between the force $\left(\mathbf{w}_{2}\left(\mathbf{w}_{4}\right)\right)$ generated at the same point of the two RPU limbs and the velocity $\left(\mathbf{Q w} \mathbf{w}_{4}\left(-\mathbf{Q w} \mathbf{w}_{2}\right)\right)$ of point $A$. It indicates that when the actuated joint is locked, there is transmission capability of the force/motion from limb 2(4) to limb 4(2); $\alpha$ is the angle between the $\mathbf{w}_{2}$ and $\mathbf{w}_{4}$.

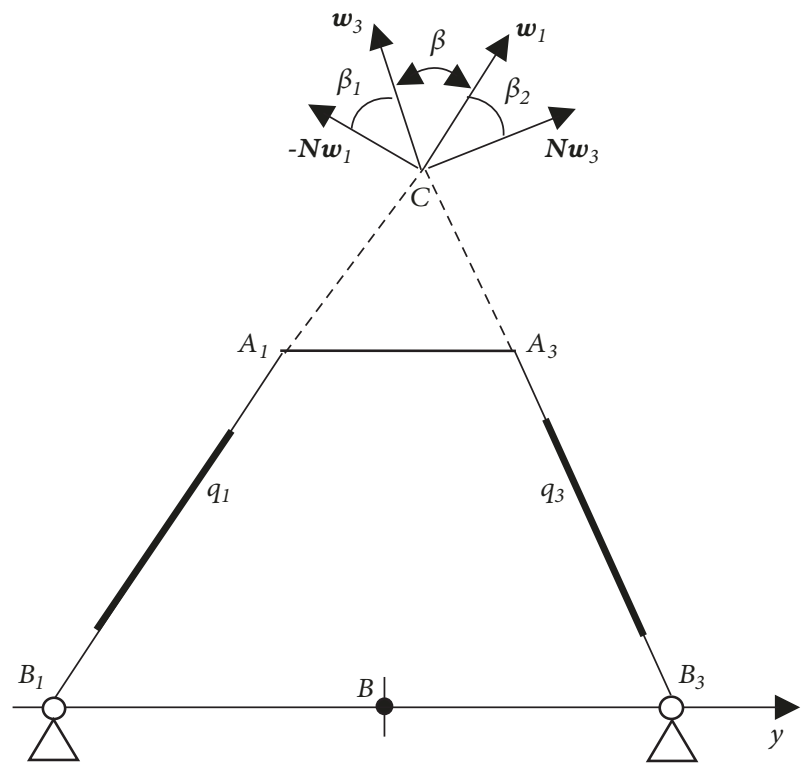

FIGURE 12: Pressure angles between SPS limbs.

Right for the SPS limb, the acute angle $\beta_{i}$ shown in Figure 12 is angle between the force $\left(\mathbf{w}_{1}\left(\mathbf{w}_{3}\right)\right)$ generated at the same point of the two SPS limbs and the velocity $\left(\mathbf{N w}_{1}\left(-\mathbf{N w}_{3}\right)\right)$ of point $C$. It indicates that when the actuated joint is locked, there is transmission capability of the force/motion from limb 1(3) to limb 3(1). $\beta$ is the angle between the $\mathbf{w}_{1}$ and $\mathbf{w}_{3}$.

For evaluating the transmission performance of the mechanism, the definitions of the transmission performance indicators are

$$
\begin{aligned}
& \lambda_{1}=\cos (\alpha), \\
& \lambda_{2}=\cos (\beta)
\end{aligned}
$$

When the transmission efficiency index $\lambda_{i}$ of the limb is closer to 1, it indicates that the transmission efficiency of the mechanism is higher. On the contrary it is relatively low, and the range of transmission efficiency index is $0 \sim 1$.

Through the research of the 2-RPU\&2-SPS SPM and the definition and analysis of the transmission performance, the Matlab software is used to simulate the transmission performance of the mechanism. According to the structural characteristics of the 2-RPU \& 2-SPS SPM, the scale parameters of the mechanism are selected as $a_{1}=a_{3}=150 \mathrm{~mm}$, $a_{2}=a_{4}=75 \mathrm{~mm}, b_{1}=b_{3}=550 \mathrm{~mm}$, and $b_{2}=b_{4}=350 \mathrm{~mm}$, and the basic working parameters are selected as $-40^{\circ} \leq$ $\psi($ deg. $) \leq 40^{\circ}, 10^{\circ} \leq \theta$ (deg. $) \leq 50^{\circ}$, and $700 \leq z(\mathrm{~mm}) \leq 900$. The simulation results shown in Figures 13 and 14.

As shown in Figures 13 and 14, the output transmission performance of each limb increases with the increase of $z$ under the condition of the scale parameter and working parameter of a given mechanism, which shows that the output transmission performance of the mechanism increases with the increase of $z$ value which is big and getting better. When the $z$ value is a certain value, the output transmission performance of the R $\underline{P} U$ limb increases with the increase 


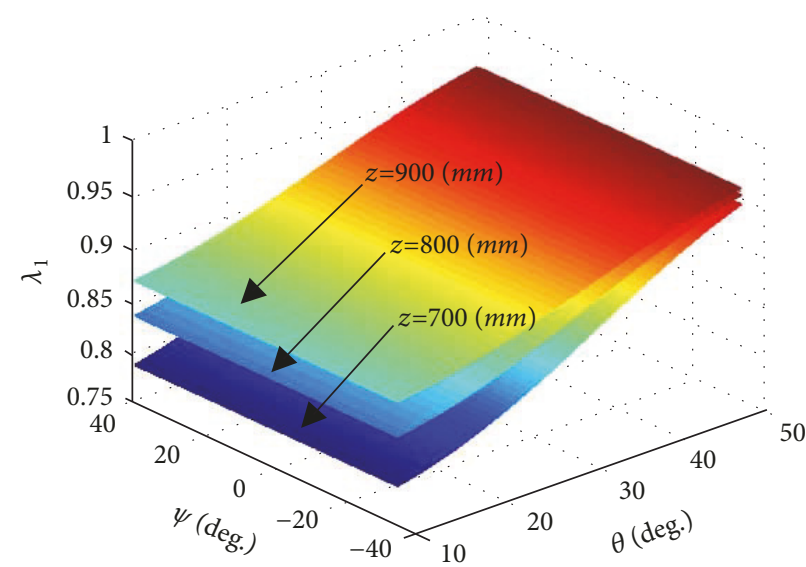

FIGURE 13: Output efficiency coefficient of the limb Rㅁ․

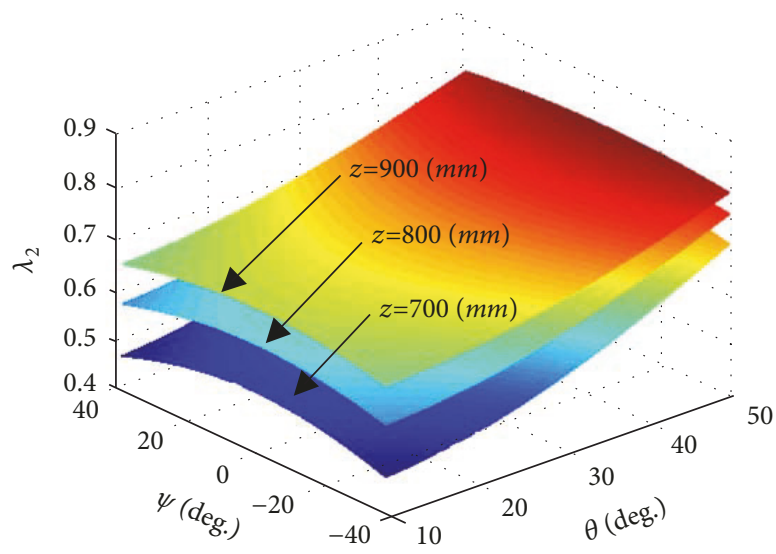

FIGURE 14: Output efficiency coefficient of the limb SPS.

of the angle $\theta$. When $\theta=10^{\circ}$, the limb output transmission performance is the worst and the output transmission performance of the limb is independent of the size of the angle $\psi$. Similarly, for the SPS limb, when the value of $z$ is certainly, the output transmission performance of the limb decreases with the increase of the angle $\psi$. when $\psi=0^{\circ}$, the output transmission performance of the limb is the best, at the same time, the output transmission performance of the limb increases with the increase of $\theta$. when $\theta=10^{\circ}$, the output transmission performance of the mechanism is the worst.

\section{Conclusions}

In this paper, the DOF of the 2-RPU\&2-SPS SPM is analyzed by using the screw theory. At the same time, the position analysis of the 2-RPU\&2-SPS SPM is obtained by utilizing the vector method, and the inverse kinematic solution of the 2-RPU\&2-SPS SPM is acquired. The forward kinematic solution of the 2-RPU\&2-SPS SPM is obtained by using the mathematical calculation software. Based on the screw theory, the entire Jacobian matrix of the 2-RPU\&2-SPSS SPM is established and the mechanism working space is studied. Finally, through the definition of output efficiency index of limb output, the kinematic transmission performance of the mechanism is evaluated by MATLAB software simulation, which provides some reference value for the optimization design and dynamic research of the follow-up mechanism.

\section{Data Availability}

The data used to support the findings of this study are available from the corresponding author upon request.

\section{Conflicts of Interest}

The authors declare that there are no conflicts of interest regarding the publication of this paper.

\section{Acknowledgments}

This work was supported by the Intelligent Manufacturing Technology Major Project of Tianjin (nos. 15ZXZNGX00270 and 16ZXZNGX00070) and the National Key Research and Development Program of China (nos. 2017 YFB1302103 and 2017YFB1303502).

\section{References}

[1] K. H. Hunt, "Structural kinematics of in-parallel-actuated robot-arms," Journal of Mechanisms, Transmissions, and Automation in Design, vol. 105, no. 4, pp. 705-712, 1983.

[2] K. J. Waldron, M. Raghavan, and B. Roth, "Kinematics of a hybrid series-parallel manipulation system," Journal of Dynamic Systems, Measurement, and Control, vol. 111, no. 2, pp. 211-221, 1989.

[3] S.-M. Song and M.-D. Zhang, "Study of reactional force compensation based on three-degree-of freedom parallel platforms," Journal of Robotic Systems, vol. 12, no. 12, pp. 783-794, 1995.

[4] Z. Huang, J. Wang, and Y. F. Fang, "Analysis of instantaneous motions of deficient-rank 3-RPS parallel manipulators," Mechanism and Machine Theory, vol. 37, no. 2, pp. 229-240, 2002.

[5] Y. Fang and Z. Huang, "Kinematics of a three-degree-offreedom in-parallel actuated manipulator mechanism," Mechanism and Machine Theory, vol. 32, no. 7, pp. 789-796, 1997.

[6] A. Sokolov and P. Xirouchakis, Kinematics of a 3-DOF Parallel Manipulator with an R-P-S Joint Structure, Cambridge University Press, 2005.

[7] J. Schadlbauer, D. R. Walter, and M. L. Husty, "The 3-RPS parallel manipulator from an algebraic viewpoint," Mechanism and Machine Theory, vol. 75, pp. 161-176, 2014.

[8] N. Farhat, V. Mata, Á. Page, and F. Valero, "Identification of dynamic parameters of a 3-DOF RPS parallel manipulator," Mechanism and Machine Theory, vol. 43, no. 1, pp. 1-17, 2008.

[9] H. Li, Z. Yang, T. Huang et al., "Dynamics and optimization of a 2-Dof parallel robot with flexible links," in Proceedings of the 7th World Congress on Intelligent Control and Automation, vol. 27, pp. 1000-1003, 2008.

[10] R. Clavel, "A fast robot with parallel geometry," in Proceedings of the International Symposium on Industrial Robots, pp. 91-100, 1988. 
[11] M. A. Hosseini, H.-R. M. Daniali, and H. D. Taghirad, "Dexterous workspace optimization of a Tricept parallel manipulator," Advanced Robotics, vol. 25, no. 13-14, pp. 1697-1712, 2011.

[12] H. T. Liu, T. Hung, J. P. Mei et al., "Kinematic design of a 5-DOF hybrid robot with large workspace/limb-stroke ratio," Journal of Mechanical Design, vol. 129, no. 5, pp. 530-537, 2006.

[13] D. Zeng, Y. Hou, Z. Huang, and W. Lu, “Type synthesis and characteristic analysis of a family of 2-DOF rotational decoupled parallel mechanisms," Chinese Journal of Mechanical Engineering, vol. 22, no. 6, pp. 833-840, 2009.

[14] Q. Li and J. M. Hervé, "Type synthesis of 3-DOF RPR-equivalent parallel mechanisms," IEEE Transactions on Robotics, vol. 30, no. 6, pp. 1333-1343, 2017.

[15] X. Kong, "Type synthesis of 3-DOF parallel manipulators with both a planar operation mode and a spatial translational operation mode," Journal of Mechanisms and Robotics, vol. 5, no. 4, Article ID 041015, 2013.

[16] H. Khakpour, L. Birglen, and S.-A. Tahan, "Synthesis of differentially driven planar cable parallel manipulators," IEEE Transactions on Robotics, vol. 30, no. 3, pp. 619-630, 2014.

[17] L. H. Rolland, "The manta and the kanuk: novel 4-DOF parallel mechanisms for industrial handling," in Proceedings of the ASME Dynamic Systems and Control Division, vol. 67, pp. 831844, 1999.

[18] T. S. Zhao and Z. Huang, "A novel spatial 4-DOF parallel mechanism and its position analysis," Mechanical Science and Technology, vol. 19, no. 6, pp. 927-929, 2000.

[19] P. Araujo-Gómez, V. Mata, M. Díaz-Rodríguez, A. Valera, and A. Page, "Design and kinematic analysis of a novel 3UPS/RPU parallel kinematic mechanism with $2 \mathrm{~T} 2 \mathrm{R}$ motion for knee diagnosis and rehabilitation tasks," Journal of Mechanisms and Robotics, vol. 9, no. 6, Article ID 061004, 2017.

[20] Q. Zhao, J. Mei, T. Song, and S. Liu, "Pressure angle in parallel mechanisms: from planar to spatial," Transactions of Tianjin University, vol. 22, no. 5, pp. 411-418, 2016.

[21] L. M. Zhang et al., "Dimensional synthesis of the Delta robot using transmission angle constraints Dimensional synthesis of the Delta robot using transmission angle constraints," Robotica, vol. 30, no. 3, pp. 343-349, 2012. 


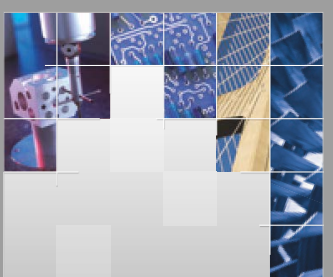

\section{Enfincering}
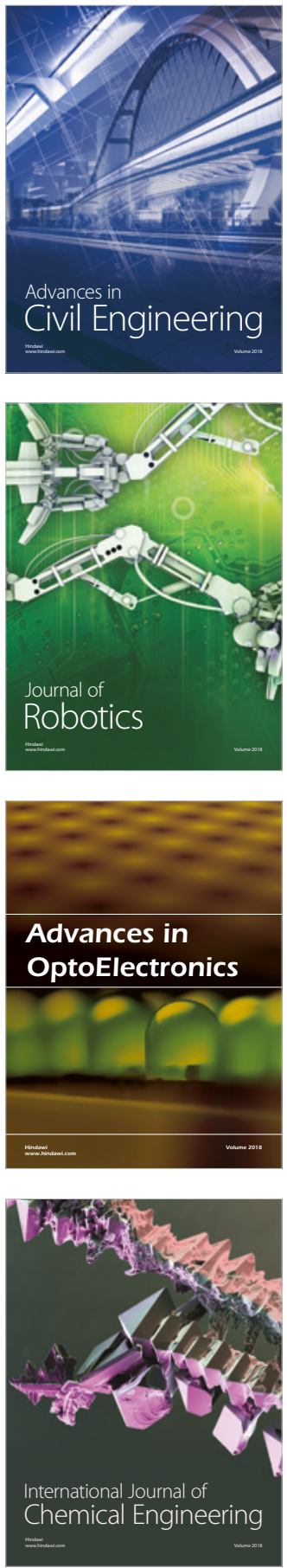

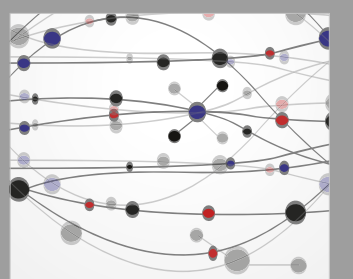

\section{Rotating \\ Machinery}

The Scientific World Journal

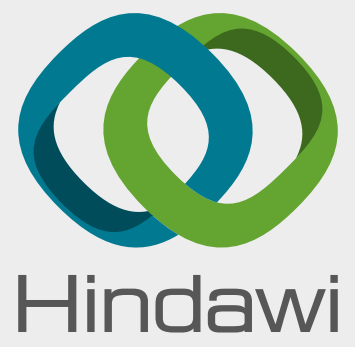

Submit your manuscripts at

www.hindawi.com
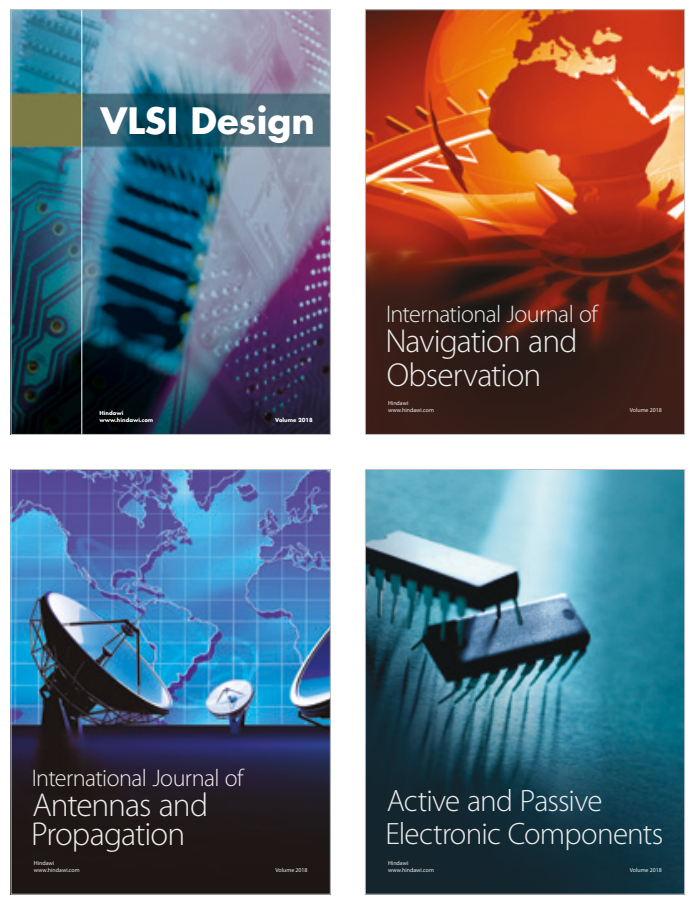
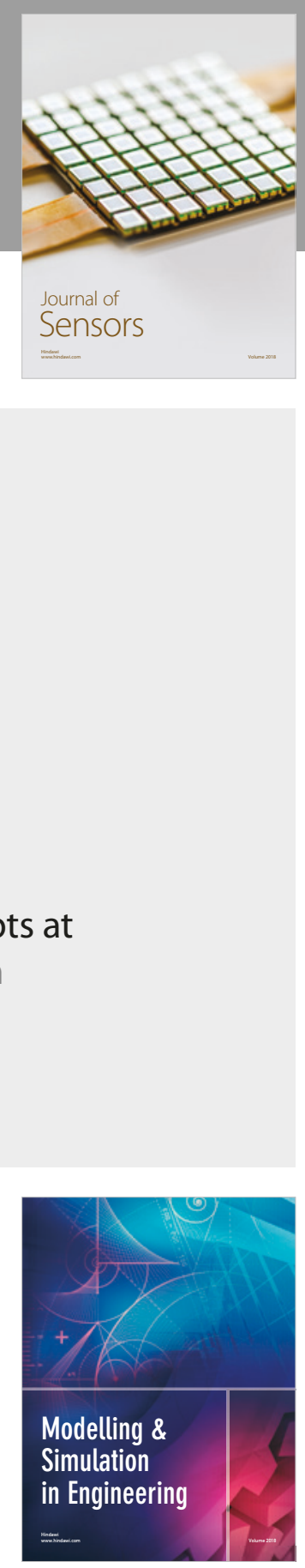

\section{Advances \\ Multimedia}
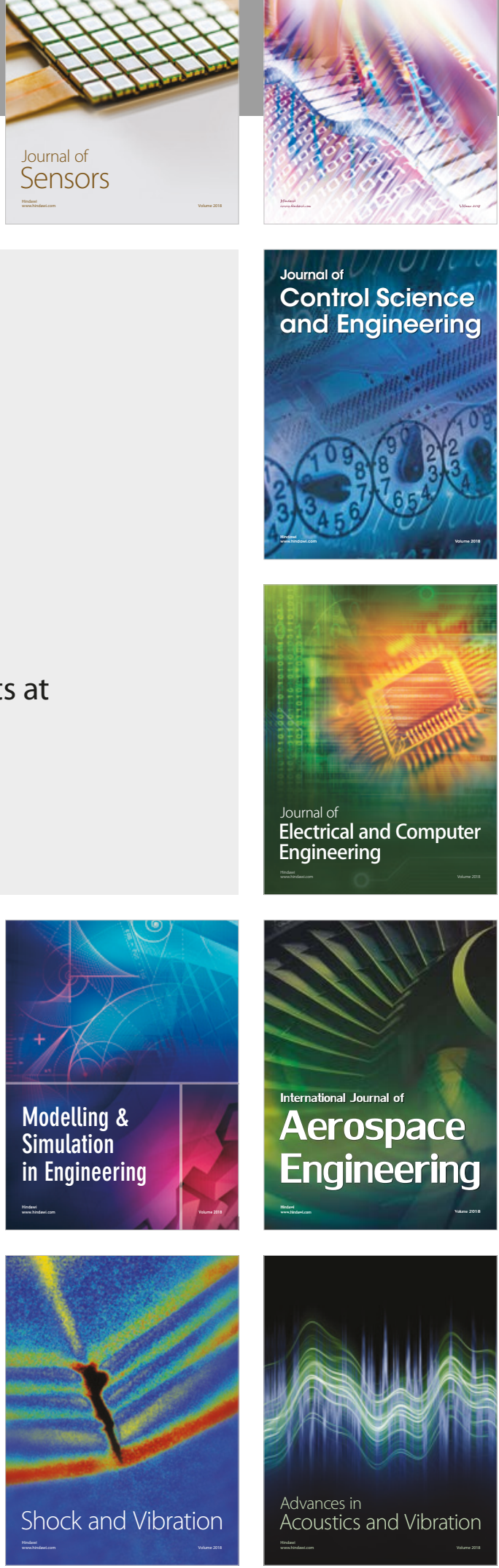